Review

\title{
A Review of Closed-Loop Algorithms for Glycemic Control in the Treatment of Type 1 Diabetes
}

\author{
Joseph El Youssef, Jessica Castle and W. Kenneth Ward ${ }^{*}$
}

Schnitzer Diabetes Center, mailstop OP05-DC, Oregon Health and Science University. 3181 SW Sam Jackson Park Road, Portland OR USA 97239

E-mails: elyoussj@ohsu.edu (J.El Y.); castleje@ohsu.edu (J.R.C.); kward@legacyglucosesensor.org, wardk@ohsu.edu (W.K.W.)

* Author to whom correspondence should be addressed: wardk@ohsu.edu (W.K.W.)

Received: 30 October 2008; in revised form: 23 January 2009 / Accepted: 25 February 2009 /

Published: 12 March 2009

\begin{abstract}
With the discovery of insulin came a deeper understanding of therapeutic options for one of the most devastating chronic diseases of the modern era, diabetes mellitus. The use of insulin in the treatment of diabetes, especially in those with severe insulin deficiency (type 1 diabetes), with multiple injections or continuous subcutaneous infusion, has been largely successful, but the risk for short term and long term complications remains substantial. Insulin treatment decisions are based on the patient's knowledge of meal size, exercise plans and the intermittent knowledge of blood glucose values. As such, these are open loop methods that require human input. The idea of closed loop control of diabetes treatment is quite different: automated control of a device that delivers insulin (and possibly glucagon or other medications) and is based on continuous or very frequent glucose measurements. Closed loop insulin control for type 1 diabetes is not new but is far from optimized. The goal of such a system is to avoid short-term complications (hypoglycemia) and long-term complications (diseases of the eyes, kidneys, nerves and cardiovascular system) by mimicking the normal insulin secretion pattern of the pancreatic beta cell.

A control system for automated diabetes treatment consists of three major components, (1) a glucose sensing device that serves as the afferent limb of the system; (2) an automated control unit that uses algorithms which acquires sensor input and generates treatment outputs; and (3) a drug delivery device (primarily for delivery of insulin), which serves as the system's efferent limb.
\end{abstract}


There are several major issues that highlight the difficulty of interacting with the complex unknowns of the biological world. For example, development of accurate continuous glucose monitors is crucial; the state of the art in 2009 is that such devices sometimes experience drift and are intended only to supplement information received from standard intermittent blood glucose data. In addition, it is important to acknowledge that an "automated" closed loop pancreas cannot approach the complexity of the normal human endocrine pancreas, which takes continuous data from substrates, hormones, paracrine compounds and autonomic neural inputs, and in response, secretes four hormones. Another major issue is the substantial absorption/action delay of insulin given by the subcutaneous route. Because of this delay, some researchers have recently given a portion of the mealrelated insulin in an open loop manner before the meal and found this hybrid approach to be superior to closed loop control.

Proportional-Integral-Derivative (PID) systems adapted from the industrial sector utilize control algorithms that alter output based on proportional (difference between actual and target levels), derivative (rate of change) and integral (time-related summative) errors in glucose. These algorithms have proven to be very promising in limited clinical trials. Related algorithms include a "fading memory" system that combines the proportionalderivative components of a classic PID system with time-relating decay of input signals that allow greater emphasis on more recent glucose values, a characteristic noted in mammalian beta-cells.

Model Predictive Control (MPC) systems are highly adaptive methods that utilize mathematical models based on observations of biological behavior patterns using system identification and are now undergoing testing in humans. The application of further mathematical models, such as fuzzy control and artificial neural networks, are also promising, but are largely clinically untested.

In summary, the prospects for closed loop control of glycemia in persons with diabetes have improved considerably. Major limitations include the delayed absorption/action of subcutaneous insulin and the imperfect stability of currently-available continuous glucose sensors. The potential for improved glycemic control in persons with diabetes brings with it the potential for reduction in the frequency of acute and chronic complications of diabetes.

Keywords: diabetes, sensor, algorithm, closed loop

\section{Introduction}

Since its discovery in 1921, insulin has proven to be one of the medical community's greatest weapons against the growing scourge of diabetes mellitus. One of the largest burdens in terms of morbidity and mortality in the Western world, its prevalence is also rising in Asia and India. Diabetes creates a huge financial burden in terms of illness and through lost work hours [1]. Acute and chronic complications of diabetes are common; diabetes is the most common cause of (a) kidney failure, (b) 
blindness in adults under the age of 65 and (c) non-traumatic limb amputation. Chronically elevated blood glucose predisposes to these chronic complications [2].

Type 1 diabetes mellitus is a disease that is characterized by autoimmune destruction of the pancreas' islet beta cells and often presents at an early age. Type 1 diabetes eventually leaves the patient completely insulin deficient, thus generating a risk for diabetic ketoacidosis. Historically, before the discovery of insulin, many patients with type 1 diabetes were confined to hospital wards with little hope of recovery from the ketoacidotic state. Later, the discovery of insulin allowed rapid recovery from comatose states [3].

Today, the management of type 1 diabetes remains a challenge, despite commercially-available recombinant human insulin and its analogs. In addition to the loss of insulin, many persons with type 1 diabetes have insufficient release of the counter-regulatory hormone glucagon, leading to a propensity for prolonged hypoglycemia during insulin treatment. This issue appears to be a greater problem in persons who suffer repeated bouts of hypoglycemia and can be improved by assiduously avoiding hypoglycemia. Due to endogenous endocrine pancreatic dysregulation, tight glycemic control with subcutaneous depot insulin regimens and subcutaneous infusion pump treatment often eludes even the most experienced diabetologist. Though these treatments have greatly enhanced diabetes care over the last few decades [2], hypoglycemia remains a very commonly encountered barrier to intensive control $[2,4]$.

Continuous insulin infusion using traditional pump technology requires input of pre-determined basal and bolus delivery rates, insulin sensitivity (correction) factors and insulin to carbohydrate ratios. Capillary glucose monitoring must be carried out four to six times per day for use as the basis of insulin dosage calculations. With such “open-loop” systems, the susceptibility to abnormally high or low glucose values is quite high. For this reason, the concept of closed loop glycemic control, in which continuous glucose monitoring is coupled to a fully automated insulin delivery device, without human input, has been an attractive idea. Patients would welcome the freedom from the need to make insulin dosing decisions many times per day. Preliminary studies using closed loop treatment have been shown to lead to improved diabetes control [5, 6].

The present article reviews the history, structure and clinical applicability of commonly used control algorithms in modern closed loop insulin-delivery systems.

\section{History of Closed Loop Systems}

Over the last half century, automated systems for delivery of insulin have been a topic of much interest, envisioned as an intelligent treatment paradigm for the insulin-deficient patient. The concept of an artificial pancreas and the evolving algorithms during this time parallel the rise of the electronic, digitized computer age. The control of blood glucose levels has been likened to industrial processes in which a monitoring system (glucose sensor) evaluates an input (glucose levels) and uses a control system (e.g. algorithm programmed into a computer) to predictably control the output of that system (insulin infusion rate, see figure 1). 
This system of artificially maintaining glycemic control requires three main components:

1. Glucose sensing device (continuous or very frequent)

2. Control device for analyzing blood glucose data and computing insulin dosing (computer or microprocessor)

3. Insulin delivery device (usually a subcutaneous mechanical pump, potentially an implantable pump)

Figure 1. Principles of control systems applied to glycemic control.

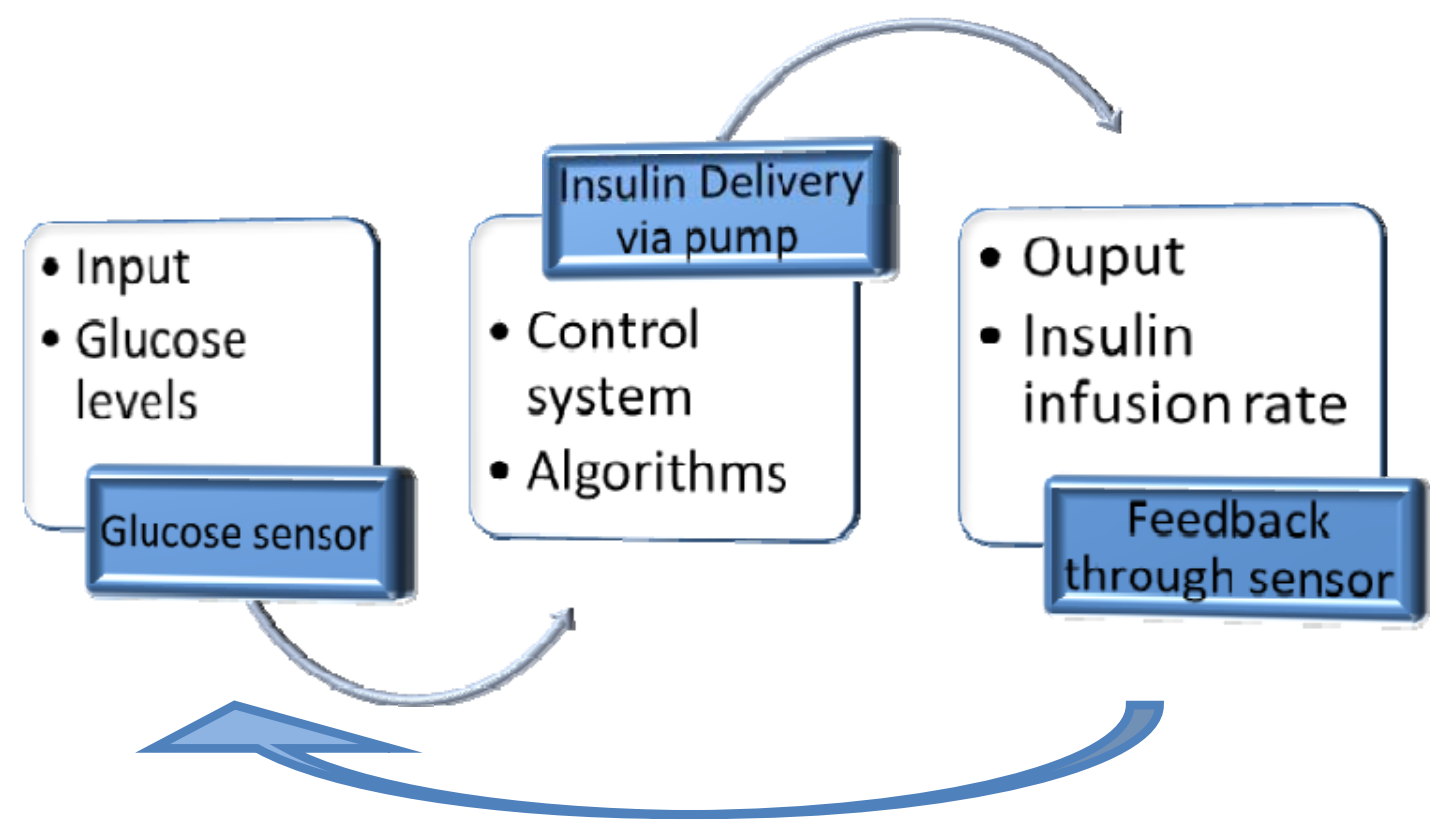

Only within the last decade have continuous glucose sensing devices (the afferent limb of a closed loop system) been approved by regulatory agencies after overcoming accuracy and stability issues. Three currently-available models which are inserted into subcutaneous tissue have been approved in the USA due to success in clinical trials (Medtronic Guardian or Paradigm, Dexcom Seven, and Abbott Navigator). A fourth, the Gluco-Day sensor, a microdialysis device (Menarini), is approved in parts of Europe. Even without closed loop control, a recent 6-month study found that usage of these devices led to a substantial improvement in hemoglobin A1C without an increase in hypoglycemic episodes in persons with Type 1 diabetes [7]. Intravenous (IV) glucose sensors have been investigated in smaller trials [8] but IV devices are not typically used. In contrast to subcutaneous sensors, intravenous sensors are accompanied by more severe risks such as thrombosis, embolization, and intravascular infection.

There have also been advances in insulin pump technology over the past 2 decades. Commerciallyavailable insulin pumps deliver insulin by the subcutaneous route, though intraperitoneal delivery (leading to faster absorption and action than subcutaneous) is being studied in limited research settings [8]. Nonetheless, subcutaneous insulin delivery does not compare favorably with the physiological secretion of beta-cells directly into the blood stream, and confers risks when used in the setting of closed-loop management. For example, delay of subcutaneous insulin absorption and action, even with 
fast-acting analog insulin, constitutes a fundamental problem of most closed loop systems in persons with diabetes: the efferent delay. Specifically, elevated glucose levels after meals due to this delay lead to late and often excessive insulin delivery, leading in turn to hypoglycemia (overcorrection hypoglycemia). Stated in engineering terminology, there is an instability characterized by large oscillations in the controlled variable (glucose) due to marked efferent delay. Recently, a "semi-closed loop" or "hybrid" system involving open-loop insulin delivery before meals has been shown to lead to tighter glycemic control than fully closed loop treatment [9]. The attractiveness of a hybrid system rests on the well-known delay between administration of subcutaneous fast-acting insulin and its action. Insulin given before the meal in this fashion can be thought of as "anticipatory" rather than reactive.

There have been multiple mathematical concepts with initial linear equations using first-order dynamics utilized in algorithms for closed loop glycemic control [10]. These early concepts could not be fully tested in animal or human studies and were rudimentary in terms of their ability to control blood glucose. Over the years, there came a clear need for models of glucose-insulin interaction that described the dynamics of carbohydrate homeostasis. A very early concept, Bolie's two-compartment model [10], gave way to Cerasi's three-compartment model [11, 12]. Later, the Bergman/Cobelli wellknown minimal model of carbohydrate metabolism was published [13], and continues to provide an important contribution to some closed loop algorithms. This model generates indices of insulin action and insulin secretion and utilizes frequent glucose and insulin measurements during glucose clamp testing or intravenous glucose tolerance testing. It assumes a closed-loop relationship between glucose, insulin secretion and insulin action between a single glucose compartment and two insulin compartments [13]. It has been instrumental in defining the disposition index, a constant product of insulin sensitivity and insulin secretion [14]. Cobelli et al have modified the original model in order to improve the accuracy of estimating insulin action and glucose secretory characteristics [15].

The ultimate role of any insulin delivery system is provision of insulin in a manner mirroring, as closely as possible, the human beta-cell. Beta-cell physiology and pathophysiology is an important topic of research and recent work emphasizes the acute effects of incretins and the chronic effects of amyloid [16]. Earlier beta cell reviews were silent on these issues and instead emphasized the many hormonal and non-hormonal input signals which regulate pancreatic insulin secretion [17].

These reviews of pancreatic beta cell secretion reveal something very important to this discussion. The strongest single regulatory factor affecting beta-cell insulin secretion is the concentration of glucose. Nonetheless, there are many other important input factors, including other pancreatic hormones, pituitary hormones, autonomic neural signals, paracrine compounds, catecholamines, other stress hormones, intestinal hormones such as glucagon-like polypeptide 1 (GLP-1), and even autocrine effects. For this reason, it is important that the reader understand how unlikely it is to achieve perfect glycemic control by using glucose concentration as the sole input for insulin delivery. Indeed, the term artificial pancreas is a presumptuous misnomer. It is impossible to perfectly replicate natural beta-cell control of insulin secretion in any one individual. Although perfection cannot be the goal, creation of an artificial pancreas (or "automated pancreas") is still an important and laudable goal, given the potential to decrease acute and chronic diabetes complications. 


\section{Proportional-Integral-Derivative (PID) Algorithms}

PID algorithms are offshoots of proportional derivative (PD) systems. A PD system from the company Nikkiso, in Japan, is used in hospital settings with continuous glucose monitoring, in order to control glucose. Garry Steil and his colleagues have been instrumental in conceptualizing and testing PID algorithms for closed loop control. Before examining their data, it will be instructive to review the fundamentals of PID control, an engineering concept which has been widely used in industrial settings $[18,19]$. Regarding closed loop treatment of diabetes, the PID control system has recently shown great success, especially when a portion of the estimated meal insulin is given before the meal. Weinzimer et al, in their recent publication, were able to show a significant difference in glycemic control between a fully-closed loop (FCL) insulin delivery system under PID control and a hybrid closed-loop (HCL) control system in which $25-50 \%$ of a calculated pre-meal insulin dose was given before meals under the same PID control (mean glucose levels: $135 \pm 45 \mathrm{mg} / \mathrm{dl}$ HCL vs $141 \pm 55 \mathrm{mg} / \mathrm{l} \mathrm{FCL}$; peak postprandial glucose levels averaged $194 \pm 47 \mathrm{mg} / \mathrm{dl} \mathrm{HCL}$ vs $226 \pm 51 \mathrm{mg} / \mathrm{dl} \mathrm{FCL}$ ) [7].

It is important to understand the three elements of the PID system. In the setting of diabetes control, it can be easily appreciated that one needs to give more insulin as glucose concentration rises (and is further away from the target). In other words, the dose of insulin is directly related to the proportional error (current glucose minus target glucose). This element is termed the proportional factor.

The derivative element is based on the rate of change. Assume that Patient A and Patient B have glucose values of $150 \mathrm{mg} / \mathrm{dl}$ (and the target is $100 \mathrm{mg} / \mathrm{dl}$ ). Also, assume the Patient A's glucose level is rising at a rate of $1 \mathrm{mg} / \mathrm{dl}$ per minute and Patient B's glucose is rising at a rate of $2 \mathrm{mg} / \mathrm{dl}$ per minute. Despite the same proportional error, Patient B, with a rate of $2 \mathrm{mg} / \mathrm{dl}$, should get a higher dose of insulin given the more rapid glucose rise. It should be noted that derivative elements are very susceptible to noise (e.g. artifactual spikes in the glucose data). For this reason, it is often preferable to use a low-pass filter for the raw data before putting it into the algorithm that computes insulin delivery based on derivative error.

The integral change is perhaps more difficult to understand and is based on glucose concentration over a time duration. Assume that two patients have a current glucose concentration of $200 \mathrm{mg} / \mathrm{dl}$ and the rate of change over the last 10 minutes is zero. Thus, their proportional and derivative factors are identical. However, Patient A has been at $200 \mathrm{mg} / \mathrm{dl}$ for 3 hours and Patient B has been at $200 \mathrm{mg} / \mathrm{dl}$ for only 20 minutes (prior to this, B's glucose level was lower). One can see that, despite insulin delivery for many hours, Patient A's glucose has not changed, i.e. this patient is proving to be quite insulin resistant. If one integrates the glucose concentration over (say) the last 5 hours, it can be seen that the integrated area is much greater for A than B, thus requiring a larger insulin dose for $\mathrm{A}$. This is the concept of the integral component.

Steil et al have argued quite convincingly that the pancreatic islet is also controlled by proportional, derivative, and integral factors [20]. Assume an abrupt step increase in glucose. The initial rapid rise in pancreatic insulin release (within the first $5-10 \mathrm{~min}$ ) is termed the first phase response and is related primarily to the rapid rate of change (derivative component). The slower rise that persists for as long as glucose is elevated is termed the second phase response and is analogous to the proportional element (if the step change is held at $250 \mathrm{mg} / \mathrm{dl}$, the second phase secretion will be greater than if the change is held at 150). It should also be noted that the pancreatic beta cell employs something like an integral 
component during second phase secretion: insulin secretion after 3 hours of elevated glucose at a fixed level is greater than insulin secretion after only 1 hour at the same glucose level. Also included in the concept of the integral component is the concept of basal insulin, a low level of insulin that continues to be secreted at normal glucose levels even in the absence of meals. Basal insulin is important for normal glucose tolerance.

While the PID concepts are relatively straightforward, system optimization can be difficult; for this reason, some degree of tuning is necessary. While experience is certainly valuable, a formal tuning process such as the Ziegler-Nichols method may be necessary [21]. Other tuning processes include the Hermite-Biehler and Cohen-Coon methods [22]. Another helpful tool in tuning a PID system is output ramping. If there are step discontinuities in outputs, the output can be gradually moved to the new specified value.

With any PID control algorithm (and its relatives: see Fading Memory Proportional Derivative [FMPD] algorithm below), an important rule is that extensive computer simulation and/or animal studies must take place before deployment in humans. Such testing includes the concept of treatment limits. For example, should PID simulation, under some test conditions, call for subcutaneous fast analog insulin at a rate of 500 units per hour, discussion with experienced, skilled clinicians would reveal that such a large infusion rate is irrational and could likely lead to profound hypoglycemia (e.g. coma, seizure or death). Several choices present themselves at this juncture: create a limit on the insulin delivery rate or change the settings of the algorithm so that such very high insulin rates are never called for. The concept of creating a mathematical algorithm that mimics human experts in the field is known as creating an "expert system.” The reasoning power of an expert human brain should not be underestimated. Using a computer does not in any way ensure superiority to the human brain, though there are two advantages to the computer: (1) given a set of inputs, it will calculate the output exactly the same way with the same result every time, and (2) it can create an output based on programmed calculations based on complex information obtained from many sources. Despite these advantages of computers, our clinical studies philosophy is that a trained diabetes specialist should be at the bedside for all closed loop control studies in persons with diabetes, at least until initial studies have been completed and validated.

\section{Model Predictive Control Algorithms}

Also referred to as moving horizon or receding horizon control, model predictive control (MPC) is a form of control in which the current control action is obtained by solving, at each sampling point, a finite horizon optimal control problem, using the current state of the system as the initial state. This differs considerably from classic PID or other conventional algorithms that use offline control law computation; MPC systems allow parameter optimization while the algorithm is on-line and running. As a result, in a real-time continuous fashion, an MPC algorithm identifies solutions for control problems for the current state of the system and at every unit time interval, rather than providing a single solution for all points along the timeline. Also, using a finite horizon helps to provide the optimal solution for the control problem in a reasonable time frame, rather than using infinite horizon prediction, such as employed in H-infinity systems [23]. These characteristics permit MPC to control 
systems with long time delays and open-loop qualities, and these characteristics are quite relevant to the management of insulin delivery for glycemic control in subjects with type 1 diabetes [24].

Figure 2. Model Predictive Control: The graph shows the change in actual glucose, the model-predicted change in glucose and the insulin infusion rates during three horizon segments. Upon reaching the first horizon actual glucose fails to decline to the predicted target, thus the insulin infusion rate is increased (for example by lowering the estimate for insulin sensitivity). At the second horizon, glucose has declined below the target, thus insulin infusion rate is reduced. At the third horizon on the actual glucose has reached the predicted target value and the estimate of insulin sensitivity remains unchanged.

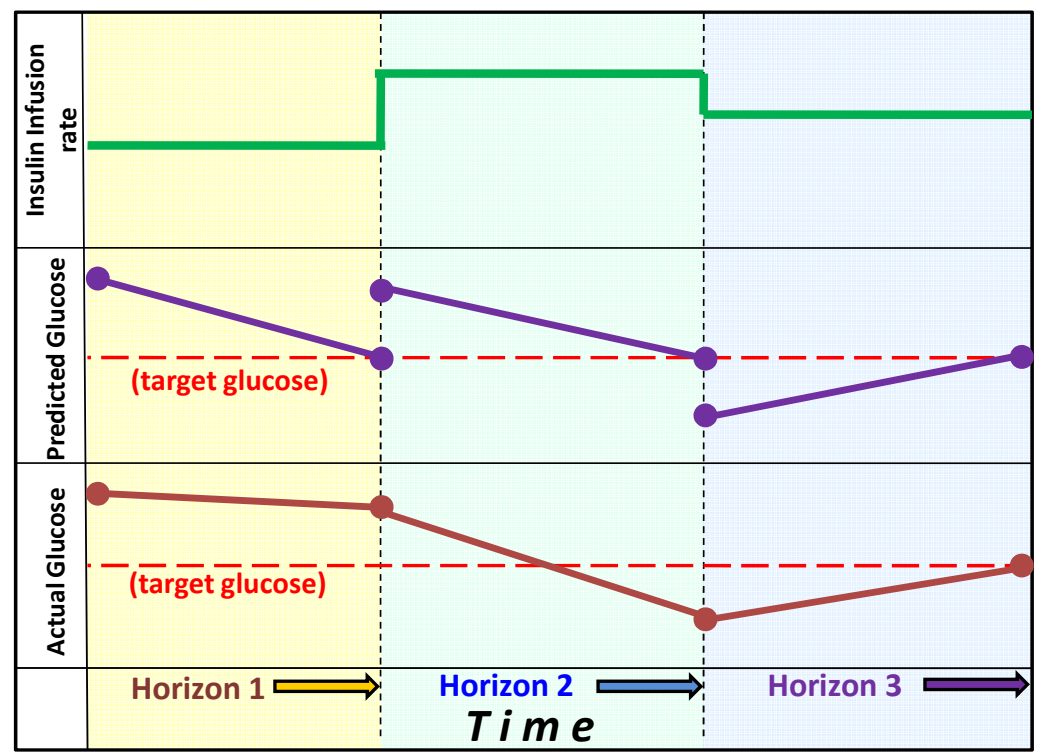

Hovorka et al developed a compartment model of the glucoregulatory system including subsystem models of carbohydrate absorption from the gut and insulin absorption subcutaneously. The controller uses Bayesian parameter estimation to determine time-varying model parameters. A moving target trajectory facilitates slow, controlled normalization of elevated glucose levels and faster normalization of low glucose values [23]. The Advanced Insulin Infusion using a Control Loop (ADICOL) project [5] initially tested the MPC algorithms in an 'in silico' environment in the hopes of developing a treatment system for subjects with type 1 diabetes. In a randomized, controlled trial of 60 patients studied during cardiac surgery in surgical care units across Europe, continuous insulin infusion therapy was evaluated under control of protocol-specific guidelines versus an MPC algorithm. The MPC algorithm showed greater ability to maintain glycemic control [24]. This trial provides promising and encouraging results that suggest the potential for safe and efficient glycemic control in an intensive care setting. Further trials in both surgical and medical intensive care settings have provided similar results with MPC algorithm-controlled glucose systems [25]. The intensive care unit is an attractive location for such studies, given the close oversight of patients/subjects by health care personnel. 
In terms of the concepts used in this and other MPC studies, it is important to note that MPC is an adaptive system and adapts itself to the input-output relationship observed during tight glucose control. That is, an incoming glucose measurement is used by the model to update model parameters such as insulin sensitivity, in view of previously administered insulin and glucose intake. MPC used in the setting of intravenous (IV) insulin delivery is simpler than the setting of subcutaneous insulin due to the fact that a submodel describing insulin absorption from the subcutaneous depot is not needed. (Nonetheless, the use of closed loop control in the hospital setting, even with IV insulin, there is marked variability among subjects in insulin sensitivity and insulin requirements based on confounding variables such as infection, physiological stress, fever, and comorbidities). The MPC glucoregulatory model determines the optimum insulin infusion rate that is expected to achieve the target glucose concentration. The outputs include insulin infusion rates that are expected, based on model predictions, to result in the target glucose concentration over a period of several hours. In is also possible to determine the frequency of glucose sampling in this method; if the glucose prediction crosses a border indicating unacceptable bounds, the MPC can suggest an additional (early) sample to be taken [24]. Glucose concentration, insulin dosage, and carbohydrate intake are some of the input variables that can be used in MPC. The insulin infusion rate and the time of the next glucose sample are the outputs in the Hovorka studies.

Kovatchev, Cobelli, and their colleagues have been instrumental in their adaptation of MPC to management of patients with type 1 diabetes by validating an MPC glucose control system utilizing an in silico model. Based upon data obtained from over 200 subjects [27], this population model was then modified to describe a patient population of persons with type 1 diabetes from which individual models of virtual patients could be extracted for running a computer simulated trial [26]. This in silico study effectively highlighted the fact that MPC systems improved glucose regulation over classical PID systems in limiting the oscillation of glucose levels. Moreover, through the recent efforts of the Juvenile Diabetes Research Foundation (JDRF), the University of Virginia and the Universities of Padova, Pavia and Montpellier, an in silico simulator for testing closed-loop algorithms is being used by the JDRF Artificial Pancreas Consortium members. A description of this in silico simulator (now based on a cohort of 300 patients with diabetes, including adults, adolescents and children) was just recently published in 2009 [27] and it has been accepted by the US Food and Drug Administration as a substitute to animal trials in the preclinical testing of closed-loop control strategies.

For more information about the mathematics of MPC systems, including many non-medical applications, we recommend to the reader a review by Mayne et al [28].

\section{Fading Memory Proportional Derivative Algorithm}

Our group has investigated an algorithm that is related to the PID control system, without the integration component. Instead of using integration to arrive at a basal and duration-related insulin delivery rates, it applies a "fading memory" of the proportional and derivative components in order to utilize the time duration of abnormal glycemia. For example, in the setting of prolonged elevation of glucose level, the algorithm does not integrate a glucose area. Instead, it applies a decay calculation so that the more recent information has a greater effect on the output than more remote information. In so doing, if glucose levels are found to be elevated over several hours, insulin delivery is increased to a 
higher rate than if glucose had been elevated only for a few minutes. However, a prolonged glucose level elevation that occurred two days previously is not relevant and does not increase the insulin delivery rate, due to the fact that the memory has completely "faded". This method has been applied to rats with type 1 diabetes with good success [29, 30]. During in vivo studies, there is a "priming" effect indicating that the pancreatic beta cell keeps a memory of prior glycemic levels, though that memory clearly fades over time [31].

Figure 3. A comparison of insulin infusion rates based only on the proportional error (IIR, PE) vs IIR based on a fading memory of PE (IIR, FMPE). When glucose is elevated to 200, note that if the IIR is based only on PE, the IIR remains steady throughout the duration of elevated glucose; but the IIR based on fading memore of PE adapts by continuing to increase the IIT during the period of hyperglycemia. The FMPD algorithm also adjusts the IIR according to a fading memory of the derivative error (not shown here). Also not shown are the basal infusion rates and glucagon infusions (using different gain factors) based on fading memories of proportional and derivative errors.

\section{Proportional Error vs Fading Memory Proportional Error}

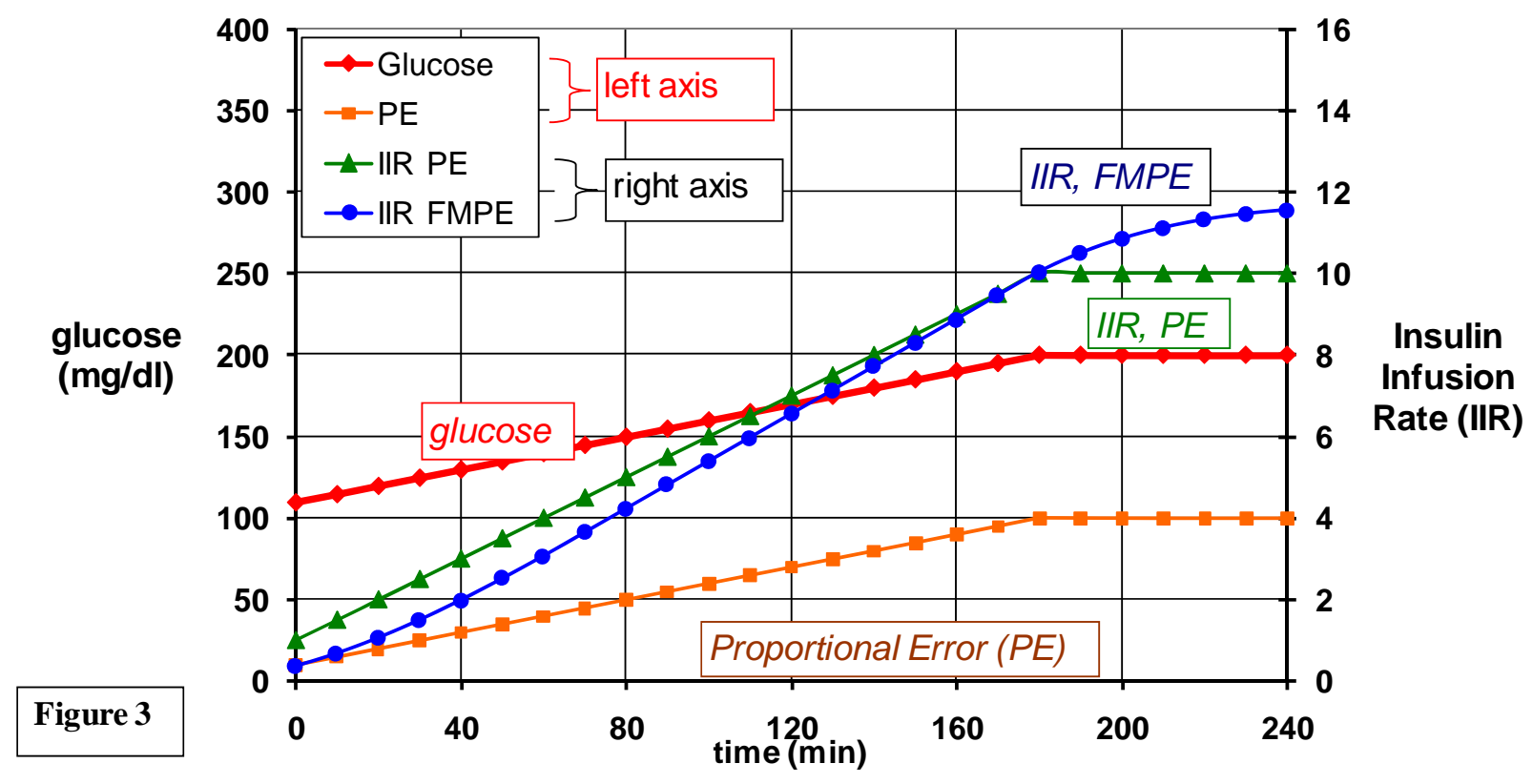

Using an FMPD approach, our group found that in animals, the use of intravenous insulin was remarkably successful in controlling glycemia [30]. When subcutaneous insulin with FMPD was used instead, the glycemic control was also successful [29], though less optimal than with IV insulin. The efferent delay of fast-acting analog insulin is assumed to be the explanation for this difference.

The following section gives the specific FMPD algorithm equations that our group used for computing insulin delivery in a recent closed loop study in rats with type 1 diabetes [32]. 
The definitions/abbreviations used in FMPD calculations are as follows:

1. $\mathrm{PE}=$ proportional error, equal to the glucose level minus the goal glucose

2. $\mathrm{DE}=$ derivative error (the "goal" derivative is zero, so the derivative is equal to the derivative error).

3. Kpe = gain coefficient for proportional error.

4. Kde = gain coefficient for derivative error.

5. Wpe $=$ weight given to a proportional error.

6. Wde $=$ weight given to a derivative error.

7. termpe $=$ weighted proportional error term that includes the gain coefficient factor .

8. termde $=$ weighted derivative error term that included the gain coefficient factor.

9. Zpe $=$ steepness coefficient for proportional error history.

10. $Z$ de $=$ steepness coefficient for derivative error history.

11. $\mathrm{t}=$ time. (Note that a $\mathrm{t}$ of 10 indicates 10 minutes back into history.)

The fundamental equation utilized by the FMPD is:

Insulin infusion rate $=$ SUM of all termpe values over the history segment + SUM of all termde values over the same history segment

90 minutes was used as the duration of the history segment. PE and DE were measured and calculated every 5 minutes. At the current time:

term $_{\mathrm{pe} 0}=\mathrm{K}_{\mathrm{pe}} * \mathrm{PE}_{0} * \mathrm{Wpe}_{0}$ term de $0=\mathrm{K}_{\mathrm{de}} * \mathrm{DE}_{0} * \mathrm{Wde} \mathrm{e}_{0}$

5 minutes back into history:

term $_{\text {pe } 5}=\mathrm{K}_{\mathrm{pe}} * \mathrm{PE}_{5} * \mathrm{Wpe}_{5} \quad$ term $_{\mathrm{de} 5}=\mathrm{K}_{\mathrm{de}} * \mathrm{DE}_{5} * \mathrm{Wde}_{5}$

The weight factors are defined as follows:

$\mathrm{W}_{\mathrm{pe}}=\mathrm{e}^{\left(-\mathrm{Zpe} \mathrm{e}^{*} \mathrm{t}\right)} \quad \mathrm{W}_{\mathrm{de}}=\mathrm{e}^{\left(-\mathrm{Zde}{ }^{*} \mathrm{t}\right)}$

Thus, for these calculations made every 5 minutes, the current insulin infusion rate is equal to:

term $_{\text {pe } 0}+$ term $_{\text {pe } 5}+$ term $_{\text {pe } 10}$, etc, $\ldots . . .+$ term pe 90

plus term de $0+$ term $_{\text {de } 5}+$ term $_{\text {de } 10}$, etc, $\ldots . . .+$ term $_{\text {de } 90}$

It can be seen that a smaller value of $\mathrm{Z}_{\mathrm{pe}}$ or $\mathrm{Z}_{\mathrm{de}}$ leads to more of the remote history being taken into consideration. Conversely, a larger value means that less of the remote history is utilized in the final

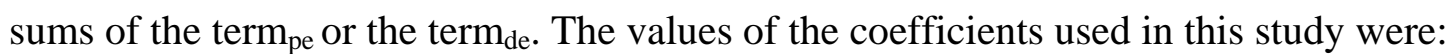

$$
\mathrm{K}_{\mathrm{pe}}: 5-7 \times 10^{-5} \quad \mathrm{~K}_{\mathrm{de}}: 0.03-0.06 \quad \mathrm{Z}_{\mathrm{pe}}: 0.025 \quad \mathrm{Z}_{\mathrm{de}}: 5 \quad \text { Glucose target: } 100 \mathrm{mg} / \mathrm{dl}
$$

It should be noted that for human use, these coefficients (intended for animal studies) may need to be modified.

In the same animal study, we found that the addition of periodic glucagon administration (given by algorithm when glucose is declining and nearing the hypoglycemic range) to continuous insulin administration is more successful in animals than insulin alone [32]. Our group is now carrying out studies in humans with insulin alone vs insulin plus glucagon in our laboratory are now underway. A group headed by Edward Damiano at Boston University has presented compelling data suggesting that (1) periodic glucagon treatment in the setting of closed loop treatment is valuable and that (2) after its reconstitution, glucagon is more stable than previously thought [33]. As an important counterregulatory hormone, glucagon is well-suited for the role proposed in the FMPD algorithm. Several studies have been able to show that glucagon significantly blunts the nadir of insulin-induced 
hypoglycemia in both in patients without diabetes and in patients with type 1 diabetes [34]. In the latter group, glucagon secretion failure often accompanies insulin deficiency, making treatment complicated as patients become prone to hypoglycemia. In our closed-loop studies, glucagon administration limited average nadirs of hypoglycemic episodes (99 $\pm 4 \mathrm{mg} / \mathrm{dl}$ with insulin plus glucagon versus $82 \pm 6$ with insulin alone) [28]. This is achieved by the fast action of subcutaneously-administered glucagon, which increases blood glucose levels within minutes of administration.

\section{Other Algorithms}

Additional systems are currently being investigated using computer models for blood glucose control. H-infinity loops attempt to minimize the worst-case gain in insulin delivery by altering insulin infusion rates from a set of pre-determined basal rates based on glucose values estimated from noisy measurements. Simulation studies showed that the system could correct blood glucose deviation using clinically acceptable insulin delivery rates, with the surmised advantage of reducing insulin requirements and the effects of hyperinsulinemia [35]. However, this method employs mathematical models that are very complex, requiring expert guidance in their management.

Fuzzy logic, on the contrary, has been used since its conception in the latter part of the last century to assist with the processing of large amounts of data by allowing 'partial set membership' rather than crisp ‘membership' or 'no membership’ [36]. Using this graded membership for given variables, and defining interaction within and between them (i.e. fuzzification of crisp values), a logic circuit for evaluation of input is created that flows through the 'fuzzy filter'. Defuzzification provides crisp output data that determines the response of the system to input analysis, generating feedback to the system that can then be re-analyzed through the loop [37]. The advantage of fuzzy control systems lies in their ability to simplify input recognition and analysis using graded, or 'fuzzied', parameters. Multiple input values therefore add minimal complexity overall, but increase stability considerably [38], inherently creating fail-safes that prevent wide fluctuations in the system. These systems have found wide applicability in other aspects of the medical field, including electro-diagnosis, control of blood pressure during anesthesia and nutrition advice.

Neural networks have come into play as key components of insulin-delivery control systems within the last few decades, not only as adjunctive components (e.g. assisting in model analysis) but also as the algorithm processor itself [39]. The adaptive capability of neural networks has been assessed within and without the medical literature, possessing remarkable self-learning and self-improving potential. In conjunction with other control systems, as shown in a randomized prospective trial of 40 critically ill patients with diabetes [40], this potential may be realized in the setting of glucoregulation. Hypoglycemic rates were almost half as common using a fuzzy-neural method of control compared to conventional algorithms, with significant improvement in glycemic control overall.

In summary, it is now possible to program the thought processes of experts into a controller that regulates the output of an insulin delivery system based on frequently-acquired glucose concentration data. The more convenient route of insulin administration (subcutaneous) leads to a substantial efferent delay due to its delayed absorption and action. For this reason, glucose levels are often elevated after meals and often too low at later periods when the insulin finally exerts this effect. In subcutaneous systems, the effect of post-prandial hyperglycemia can be mitigated by giving some of the insulin in an 
open loop fashion before the meal. In addition, the late episodes of hypoglycemia can likely be minimized by the delivery of small doses of glucagon as glucose falls towards low levels. Given the personal and economic burden of diabetes mellitus, closed loop (or hybrid) systems hold great promise for reducing its acute and chronic complications.

\section{Acknowledgements}

We gratefully acknowledge financial support from the Juvenile Diabetes Research Foundation (grant number 22-2006-1121), by the American Diabetes Association (grant number 1-R03-RA-87), and the Good Samaritan Foundation, Portland OR. We also thank Julia Engle and Ryan Massoud for excellent technical support.

\section{References and Notes}

1. American Diabetes Association. Economic costs of diabetes in the US in 2007. Diabetes Care 2008, 31, 1-20.

2. Diabetes Control and Complications Research Group. The effect of intensive treatment of diabetes on the development and progression of long-term complications in insulin-dependent diabetes mellitus. N Engl J Med 1993, 329(14), 977-86.

3. Banting, F.G.; Best, C.H.; Collip, J.B.; Campbell, W.R.; Fletcher, A.A. Pancreatic extracts in the treatment of diabetes mellitus: preliminary report. 1922. Cmaj 1922, 145(10), 1281-6, (reprinted 1991).

4. Cryer, P.E. Hypoglycaemia: the limiting factor in the glycaemic management of Type I and Type II diabetes. Diabetologia 2002, 45(7), 937-48.

5. Hovorka, R.; Chassin, L.J.; Wilinska, M.E.; Canonico, V.; Akwi, J.A.; Federici, M.O.; MassiBenedetti, M.; Hutzli, I.; Zaugg C.; Kaufmann, H.; Both, M.; Vering, T.; Schaller, H.C.; Schaupp, L.; Bodenlenz, M.; Pieber, T.R. Closing the loop: the adicol experience. Diabetes Technol Ther 2004, 6(3), 307-18.

6. Farmer Jr., T.G.; Edgar, T.F.; Peppas, N.A. The future of open- and closed-loop insulin delivery systems. J Pharm Pharmacol 2008, 60(1), 1-13.

7. Tamborlane, W.V.; Beck, R.W.; Bode, B.W.; Buckingham, B.; Chase, H.P.; Clemons, R.; FialloScharer, R.; Fox, L.A.; Gilliam, L.K.; Hirsch, I.B.; Huang, E.S.; Kollman, C.; Kowalski, A.J.; Laffel, L.; Lawrence, J.M.; Lee, J.; Mauras, N.; O'Grady, M.; Ruedy, K.J.; Tansey, M.; Tsalikian, E.; Weinzimer S.; Wilson, D.M.; Wolpert, H.; Wysocki, T.; Xing, D. Continuous glucose monitoring and intensive treatment of type 1 diabetes. $N$ Engl J Med 2008, 359(14), 1464-76.

8. Renard, E.; Costalat, G.; Chevassus, H.; Bringer, J. Artificial beta-cell: clinical experience toward an implantable closed-loop insulin delivery system. Diabetes Metab 2006, 32(5 Pt 2), 497-502.

9. Weinzimer, S.A.; Steil, G.M.; Swan, K.L.; Dziura, J.; Kurtz, N.; Tamborlane, W.V. Fully automated closed-loop insulin delivery versus semiautomated hybrid control in pediatric patients with type 1 diabetes using an artificial pancreas. Diabetes Care 2008, 31(5), 934-9.

10. Bolie, V.W. Coefficients of normal blood glucose regulation. J Appl Physiol 1961, 16, 783-8.

11. Cerasi, E.; Fick, G.; Rudemo, M. A mathematical model for the glucose induced insulin release in man. Eur J Clin Invest 1974, 4(4), 267-78. 
12. Cerasi, E. An analogue computer model for the insulin response to glucose infusion. Acta Endocrinol (Copenh) 1967, 55(1), 163-83.

13. Bergman, R.N.; Phillips, L.S.; Cobelli, C. Physiologic evaluation of factors controlling glucose tolerance in man: measurement of insulin sensitivity and beta-cell glucose sensitivity from the response to intravenous glucose. J Clin Invest 1981, 68(6), 1456-67.

14. Elbein, S.C.; Wegner, K.; Kahn, S.E. Reduced beta-cell compensation to the insulin resistance associated with obesity in members of caucasian familial type 2 diabetic kindreds. Diabetes Care 2000, 23(2), 221-7.

15. Vicini, P.; Caumo, A.; Cobelli, C. The hot IVGTT two-compartment minimal model: indexes of glucose effectiveness and insulin sensitivity. Am J Physiol 1997, 273(5 Pt 1), E1024-32.

16. Marchetti, P.; Dotta, F.; Lauro, D.; Purrello, F. An overview of pancreatic beta-cell defects in human type 2 diabetes: implications for treatment. Regul Pept 2008, 146(1-3), 4-11.

17. Ward, W.K.; Beard, J.C.; Porte, D. Islet B-cell function in human subjects, in Methods of Diabetes Research; Clarke, W.L., Larner, J. and S. Pohl, Eds.; Vol II, Wiley and Sons: New York, 1986; pp. 3-14.

18. El Asri, R.; Baxter, D. Process control in municipal solid waste incinerators: survey and assessment. Waste Manag Res 2004, 22(3), 177-85.

19. Yu, D.L.; Chang, T.K.; Yu, D.W. Fault tolerant control of multivariable processes using autotuning PID controller. IEEE Trans Syst Man Cybern B Cybern 2005, 35(1), 32-43.

20. Steil, G.M.; Rebrin, K.; Janowski, R.; Darwin, C.; Saad, M.F. Modeling beta-cell insulin secretion--implications for closed-loop glucose homeostasis. Diabetes Technol Ther 2003, 5(6), 953-64.

21. Cho, J.H.; Sung, S.W.; Lee, I.B. Cascade control strategy for external carbon dosage in predenitrifying process. Water Sci Technol 2002, 45(4-5), 53-60.

22. Roy, A.; Iqbal, K. PID controller tuning for the first-order-plus-dead-time process model via Hermite-Biehler theorem. ISA Trans 2005, 44(3), 363-78.

23. Hovorka, R.; Canonico, V.; Chassin, L.J.; Haueter, U.; Massi-Benedetti, M.; Orsini Federici, M.; Pieber, T.R.; Schaller, H.C.; Schaupp, L.; Vering, T.; Wilinska, M.E. Nonlinear model predictive control of glucose concentration in subjects with type 1 diabetes. Physiol Meas 2004, 25(4), 90520.

24. Hovorka, R.; Kremen, J.; Blaha, J.; Matias, M.; Anderlova, K.; Bosanska, L.; Roubicek, T.; Wilinska, M.E.; Chassin, L.J.; Svacina, S.; Haluzik, M. Blood glucose control by a model predictive control algorithm with variable sampling rate versus a routine glucose management protocol in cardiac surgery patients: a randomized controlled trial. J Clin Endocrinol Metab 2007, 92(8), 2960-4.

25. Pachler, C.; Plank, J.; Weinhandl, H.; Chassin, L.J.; Wilinska, M.E.; Kulnik, R.; Kaufmann, P.; Smolle, K.H.; Pilger, E.; Pieber, T.R.; Ellmerer, M.; Hovorka, R. Tight glycaemic control by an automated algorithm with time-variant sampling in medical ICU patients. Intensive Care Med 2008, 34(7), 1224-30.

26. Magni, L.; Raimondo, D.; Bossi, L.; Man, C.; Kovatchev, B.; Cobelli, C. Model Predictive Control of Type 1 Diabetes: An in Silico Trial. J Diabetes Sci and Technology 2007, 1, 804-812. 
27. Kovatchev, B.; Breton, M.; Man, C.; Cobelli, C. In Silico Preclinical Trials: A Proof of Concept in Closed-Loop Control of Type 1 Diabetes. J Diabetes Sci and Technology 2009, 3, 44-55.

28. Mayne, D.; Rawlings, J.; Rao, C.; Scokaert, M. Constrained model predictive control: Stability and optimality. Automatica 2000, 36, 789-814.

29. Gopakumaran, B.; Duman, H.M.; Overholser, D.P.; Federiuk, I.F.; Quinn, M.J.; Wood, M.D.; Ward, W.K. A novel insulin delivery algorithm in rats with type 1 diabetes: the fading memory proportional-derivative method. Artif Organs 2005, 29(8), 599-607.

30. Ward, W.K.; Wood, M.D.; Casey, H.M.; Quinn, M.J.; Federiuk, I.F. An Implantable Subcutaneous Glucose Sensor Array in Ketosis-prone Rats: Closed Loop Glycemic Control. Artif Organs 2005 29, 131-43.

31. Ward, W.K.; Bolgiano, D.C.; McKnight, B.; Halter, J.B.; Porte, D. Jr. Diminished B cell secretory capacity in patients with noninsulin-dependent diabetes mellitus. J Clin Invest 1984, 74(4), 131828.

32. Ward, W.K.; Engle, J.; Duman, H.M.; Bergstrom, C.P.; Kim, S.F.; Federiuk, I.F. The Benefit of Subcutaneous Glucagon During Closed-Loop Glycemic Control in Rats With Type 1 Diabetes. IEEE Sensors 2008, 8, 89-96.

33. El-Khatib, F.H.; Jiang, J.; Gerrity, R.G.; Damiano, E.R. Pharmacodynamics and stability of subcutaneously infused glucagon in a type 1 diabetic Swine model in vivo. Diabetes Technol Ther 2007, 9(2), 135-44.

34. Taborsky Jr., G.J.; Ahren, B.; Mundinger, T.O.; Mei, Q.; Havel, P.J. Autonomic mechanism and defects in the glucagon response to insulin-induced hypoglycaemia. Diabetes Nutr Metab 2002, 15(5), 318-22; discussion 322-3.

35. Kienitz, K.H.; Yoneyama, T. A robust controller for insulin pumps based on H-infinity theory. IEEE Trans Biomed Eng 1993, 40(11), 1133-7.

36. Kaehler, S.D. Fuzzy Logic Tutorial. Seattle Robotics Society website:

http://www.seattlerobotics.org/encoder/Mar98/fuz/flindex.html, 2008. accessed on October 28, 2008.

37. Trajanoski, Z.; Wach, P. Fuzzy filter for state estimation of a glucoregulatory system. Comput Methods Programs Biomed 1996, 50(3), 265-73.

38. Campos-Delgado, D.U.; Hernandez-Ordonez, M.; Femat, R.; Gordillo-Moscoso, A. Fuzzy-based controller for glucose regulation in type-1 diabetic patients by subcutaneous route. IEEE Trans Biomed Eng 2006, 53(11), 2201-10.

39. El-Jabali, A.K. Neural network modeling and control of type 1 diabetes mellitus. Bioprocess Biosyst Eng 2005, 27(2), 75-9.

40. Dazzi, D.; Taddei, F.; Gavarini, A.; Uggeri, E.; Negro, R.; Pezzarossa, A. The control of blood glucose in the critical diabetic patient: a neuro-fuzzy method. J Diabetes Complications 2001, 15(2), 80-7.

(C) 2009 by the authors; licensee Molecular Diversity Preservation International, Basel, Switzerland. This article is an open-access article distributed under the terms and conditions of the Creative Commons Attribution license (http://creativecommons.org/licenses/by/3.0/). 